\title{
Updating on Neuroendocrine Tumors New Clinical Evidence in the Search of the Ideal Treatment Sequence
}

\author{
Juan Manuel O'Connor* \\ Chief of the Gastrointestinal Tumor Division, Alexander Fleming Institute Neuroendocrine Tumor Department, Gastroenterology Hospital Bonorino \\ Udaondo
}

Submission: March 18, 2017; Published: April 03, 2017

"Corresponding author: Juan Manuel O'Connor, Chief of the Gastrointestinal Tumor Division, Alexander Fleming Institute Neuroendocrine Tumor Department, Gastroenterology Hospital Bonorino Udaondo Assistant Professor of Clinical Pharmacology, CEMIC University Institute, Argentina, Email: juanmanuel.oconnor@gmail.com

\section{Introduction}

Neuroendocrine tumors (NETs) represent a heterogeneous clinical entity with an increasing incidence as shown by the different registers available. As opposed to other solid tumors, NETs represent a challenge, not only from the diagnostic standpoint but also in terms of treatment strategies. According to the data published by our Working group, ARGENTUM GROUP [1], with more than 600 patients assessed, roughly $60 \%$ of the cases present with metastatic disease from the onset.

The reason why the incidence of advanced disease is high since early onset may be due to a bias, for most of the physicians in our Group are oncologists. Moreover, about $20 \%$ of the patients included present poorly differentiated NETs, a heterogeneous subgroup, with high risk and poor outcome according to the different series reported.

\section{Anatomic pathology and sub classification of g3 tumors}

$\mathrm{G}$ grading, in the classification of neuroendocrine tumors has been proposed by ENETs to identify three categories according to the proliferative value, Ki 67: Ki 67 under2\%, G1; Ki 67 between 3 and 20\%, G2; and wth a Ki 67 over 20\%, G3.

Although for therapeutic reasons patients with well differentiated tumors were classified in the same manner, at present the cut off point for G3 has been modified. As reported by Sorbye et al. [2] 55\% is the parameter to better discriminate this group of patients, based on a retrospective series including more than 300 patients with poorly differentiated neuroendocrine tumors. Moreover, the same study showed that Ki 67 was a predictive factor of response for platinum-based chemotherapy.

As recently reported and commented by Dr. G. Rindi at the 2016 Annual ENETs Conference a different biological behavior has been described even for the so called NEC G3 tumors, that is, poorly differentiated neuroendocrine carcinomas; a sub classification has been proposed for NET G3 to better differentiate them from the real NEC G3, considering that the former present more favorable characteristics for a good outcome, and even the potential somatostatin receptor expression in some cases. This sub classification is important because it favors new treatment options at least in some G3 subgroups, although a retrospective validation is required for better staging.

In a retrospective series including 136 patients with G2 gastroenteropancreatic tumors, Milione et al, [3] the Italian group, showed a possible staging system based on morphological, proliferation features, defects in the mismatch repair system (dMMR) , CD117 expression and site of origin.

The multivariate analysis in that study showed that the factors mentioned above were independent in NEC G. Three prognostic categories were defined based on these factors in G3, a category including well differentiated tumors vs poorly differentiated tumors and Ki $67,<55 \%$ versus $>55 \%$, with a 43.6 month survival in patients with well differentiated tumors and Ki 67 between 20 and 55\%.

In the case of poorly differentiated tumors and Ki 67 ranging between 20 and 55\%, median survival was 24.5 months, and finally the group with the poorest prognosis, poorly differentiated tumors with a Ki 67 over 55\%, with a survival of 5.3 months. This study clearly shows an active area for clinical research in this subgroup of patients with limited treatment options at present.

\section{New clinical evidence, randomized studies}

At the Annual Meeting of the European Conference held in Vienna in 2015 the results of different clinical trials in neuroendocrine tumors were presented. All of them were 
randomized comparative studies, evaluating efficacy and tolerance of different treatment options. Although already available in the field of neuroendocrine tumors the confirmation of a well designed comparative clinical trial to show the advantages in terms of survival is still pending.

The RADIANT4 [4] study included 302 patients and compared the use of everolimus, (10mg /day) vs placebo in patients with a diagnosis of Grade 1, 2 advanced, progressive, well-differentiated, non-functional neuroendocrine tumors of lung or gastrointestinal origin.

The primary endpoint, progression free survival (PFS) was achieved; everolimus demonstrated a $52 \%$ reduction of progression vs the non-active treatment arm, which translated intomPFS of 11 months vs 3.9 months in favor of everolimus, which was statistically significant. The most significant fact in the study is the inclusion of neuroendocrine tumors of bronchiallung origin, where the role of m-TOR inhibitors was not clear.

TheNETTER-1trial compared the use of Lu 177 DOTATATE, (Lutathera) plus Octreotide (30mg) in patients with NETs of gastrointestinal origin and advanced disease versus Octreotide (60mg) every 28 days.

The study included 229 patients, and the primary endpoint, mPFS, was achieved, with an impressive $80 \%$ reduction in risk of disease progression in the Lutathera arm (HR 0.20 IC 95\% 0.13 $0.34)$. This result translated into an mPFS not achieved yet in the Lutathera arm, and a mPFS of 8.4 months in the Octreotide arm (60mg every 28 days). The added value of the study is that it is the first clinical trial to show favorable results of the use of $\mathrm{Lu}$ 177 , in a randomized comparative trial.

Another trial, TELESTAR [4], studied the role of TELOTRISTAT ETIPRATE, which acts by inhibiting tryptophan hydroxylase, the rate limiting enzyme in the conversion of tryptophan to serotonin. The primary endpoint of the study was to reduce symptoms associated with carcinoid syndrome such as diarrhea in patients on octreotide (30mg every 28 days) whose symptoms persisted.

The study included 135 patients, randomized to three arms; in two arms two different doses of the drug were tested, and the other arm was given placebo (in fact, these patients were on Octreotide). In both arms treated with Telotristat a $40 \%$ symptom reduction was observed, and the responses was sustained in time. Also, there was a reduction in the levels of 5HIAA, as a specific marker of carcinoid syndrome. This opens the door to a new oral treatment option for functional tumors, with carcinoid syndrome previously managed with standard doses of Octreotide.

The data in CLARINET [5] have been updated; the open phase was presented, that is, the assessment study in terms of efficacy and safety in patients originally randomized to lanreotide vs placebo in the CLARINET core study. The open phase included patients with at least stable disease in the original study after 2 years of treatment, and patients who had received placebo during theinitialrandomization and evidenced disease progression.

A total of 88 patients were included, 41 previously treated with lanreotide and 47 in the placebo arm. Thirty nine percent of the patients included had intestinal tumors, 38\% had pancreatic tumors, and $23 \%$ had tumors in other locations or of unknown origin. As for safety, patients who continued on active treatment reported less adverse effects during this open phase of the study as compared to the initial phase. The group of patients treated with lanreotide, who were previously included in the placebo arm, evidenced a higher incidence of diarrhea during the initial phase; however, no other adverse events were observed. The median PFS in the CLARINET core study was 32.8 months.

The median PFS in the open phase in the group who had not received active treatment before (placebo arm) was 14 months. The results show the antiproliferative effect and the safety profile of prolonged treatment in patients with intestinal and pancreatic neuroendocrine tumors, G1-G2 with a Ki as high as $10 \%$ with Lanreotide (120mg every 28 days).

Update on the Treatment Guidelines of NETs, ENETS (European Society of Neuroendocrine Tumors). New treatment guidelines for neuroendocrine tumors of gastroenteropancreatic origin have been published this year. It should be underlined that these Guidelines are the result of the Consensus Meeting held by the members of the Advisory Board in Europe, the United States and Latin America (Argentina and Brazil). Different topics such as location of the primary tumor and disease stage are discussed at the Consensus sessions. Also, treatment options and algorithms in liver metastatic disease and high-grade neuroendocrine tumors are also dealt with.

In the case of neuroendocrine tumors of intestinal, jejunumileum [6] origin, the role of surgery as a curative procedure is underlined, or the role a large dissection of the mesenteric lymph nodes as a palliative procedure.

The management of superior mesenteric artery involvement should be discussed by a multidisciplinary team, although resection is not always feasible in advanced disease. In terms of overall survival (OS), the role or potential benefits of surgery for primary tumors of intestinal origin in asymptomatic patients with liver metastases is still debatable. The European Society is conducting a comparative clinical trial in order to find an answer [7].

A cholecystectomy may be performed during surgery to avoid the risk of complications due to bile duct lithiasis, another topic without clear supporting evidence, but with the consensus of the participants. Another important point underscored in the new version of the Guidelines is prophylaxis against carcinoid crisis with the use of somatostatin analogs in patients with carcinoid 
syndrome. The design of Treatment Guidelines and an Algorithm for patients with neuroendocrine tumors and liver metastases has been very important, for it is the most common location for this type of tumors (Figure $1 \& 2$ ).

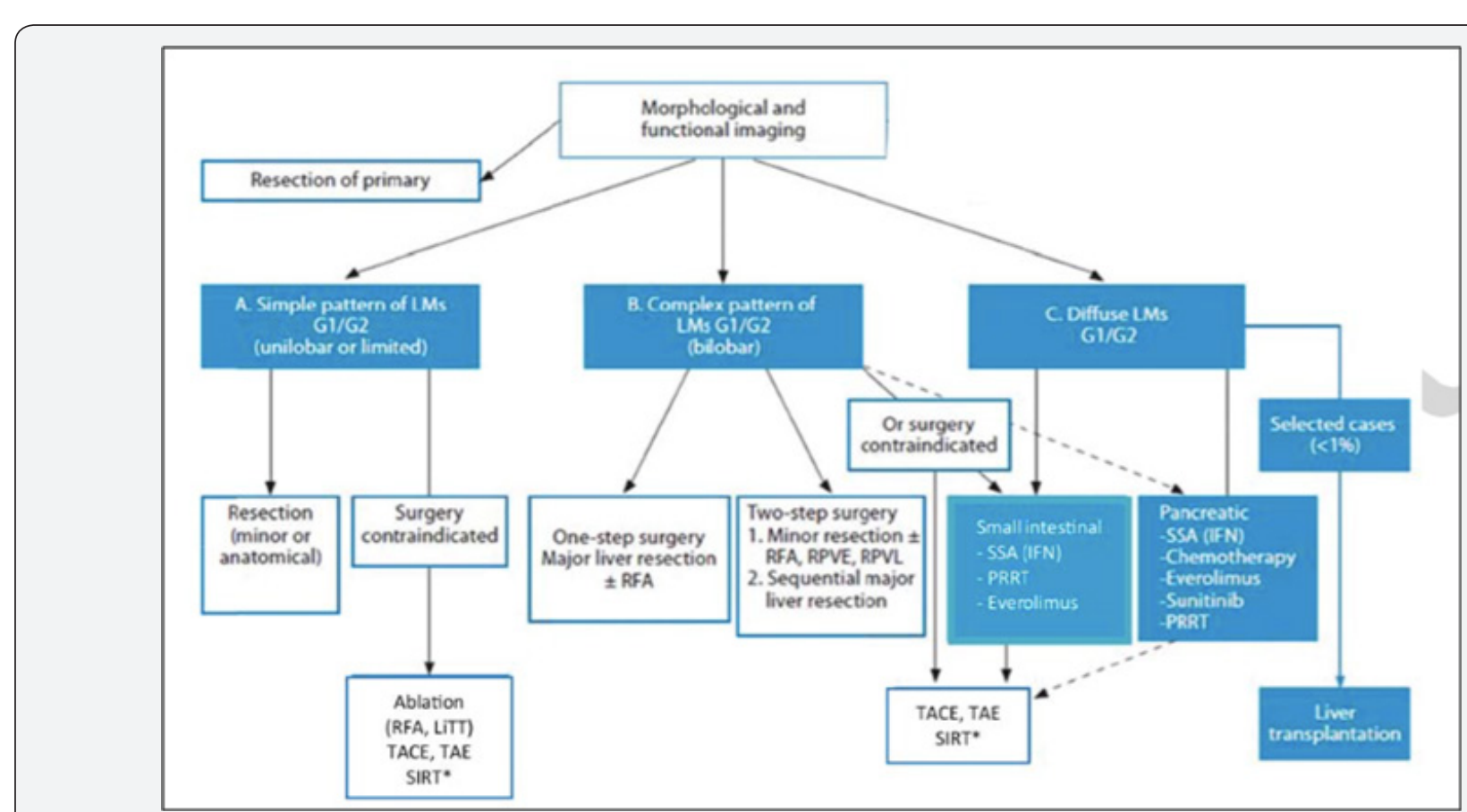

Figure 1: Algorithmfrom the ENETS7 Guidelines, Neuroendocrinology 2016.

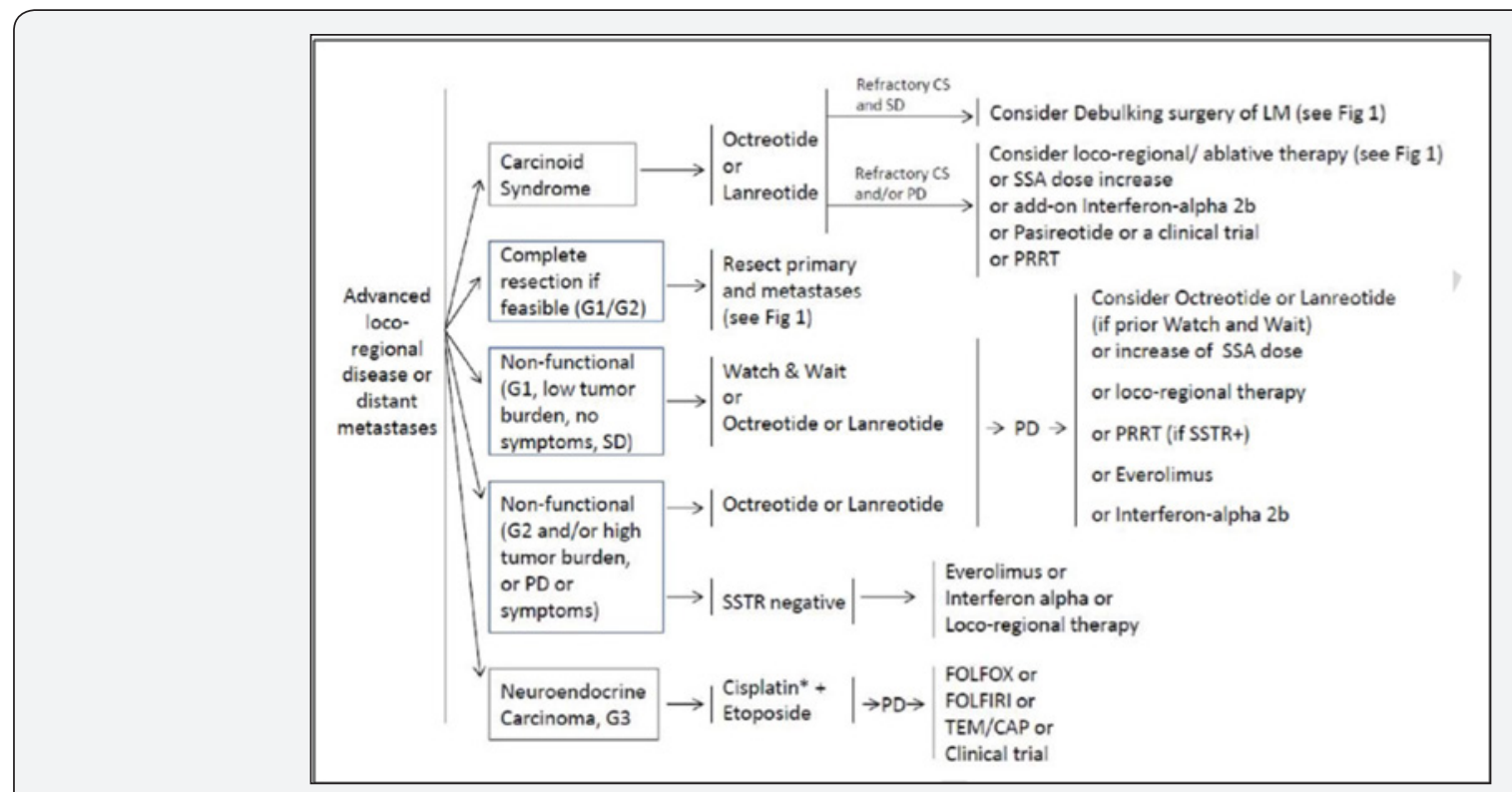

Figure 2: Algorithms proposed in patients with NETs of gastrointestinal origin and liver metastases. 2016 ENETS Guidelines.

One of the important aspects to underline is the staging system suggested by ENETS, in relation to 3 subtypes of liver involvement. In the case of a single pattern, in G1, G2, that is unilobar or restricted involvement, surgery plays a key role. In the complex or bilobar type, surgery is the option in some cases, as well as the liver regional management such as liver embolization of chemoembolization. Finally, the diffuse type is the most common clinical scenario, and is the ideal subgroup for different systemic treatment options or regional liver management, so that surgery is ruled out for this subset of patients. This subdivision may be easy in the theoretical setting but in clinical practice it is not so easy to stage patients in these three categories. Our Group is currently working on a registry of patients with liver metastases to estimate the frequency 
of presentation, in which cases surgery is performed up front, which are the treatment lines used, according to the preferences of the attending physicians, etc., in a series of cases in Argentina. Finally, the update on the treatment Guidelines also includes algorithms for neuroendocrine tumors with liver metastases, considering some differences between tumors of gastrointestinal and pancreatic origin.

In the algorithm we should underline four or five practical questions to be answered in order to define the initial treatment.
First, if the patient presents carcinoid syndrome, i.e. a functional or non functional tumor; second, is surgery as a curative procedure an option?; third, if the patient has a functional tumor, and it is a G1, with a low volume of disease and is asymptomatic; fourth, the same case as before but with a larger volume of disease and symptomatic or with progression of disease, the possibility of target therapy, such as everolimus. Finally, does the patient have a G3 tumor? In this stetting, the only option isplatinum-based chemotherapy (Figure 3).

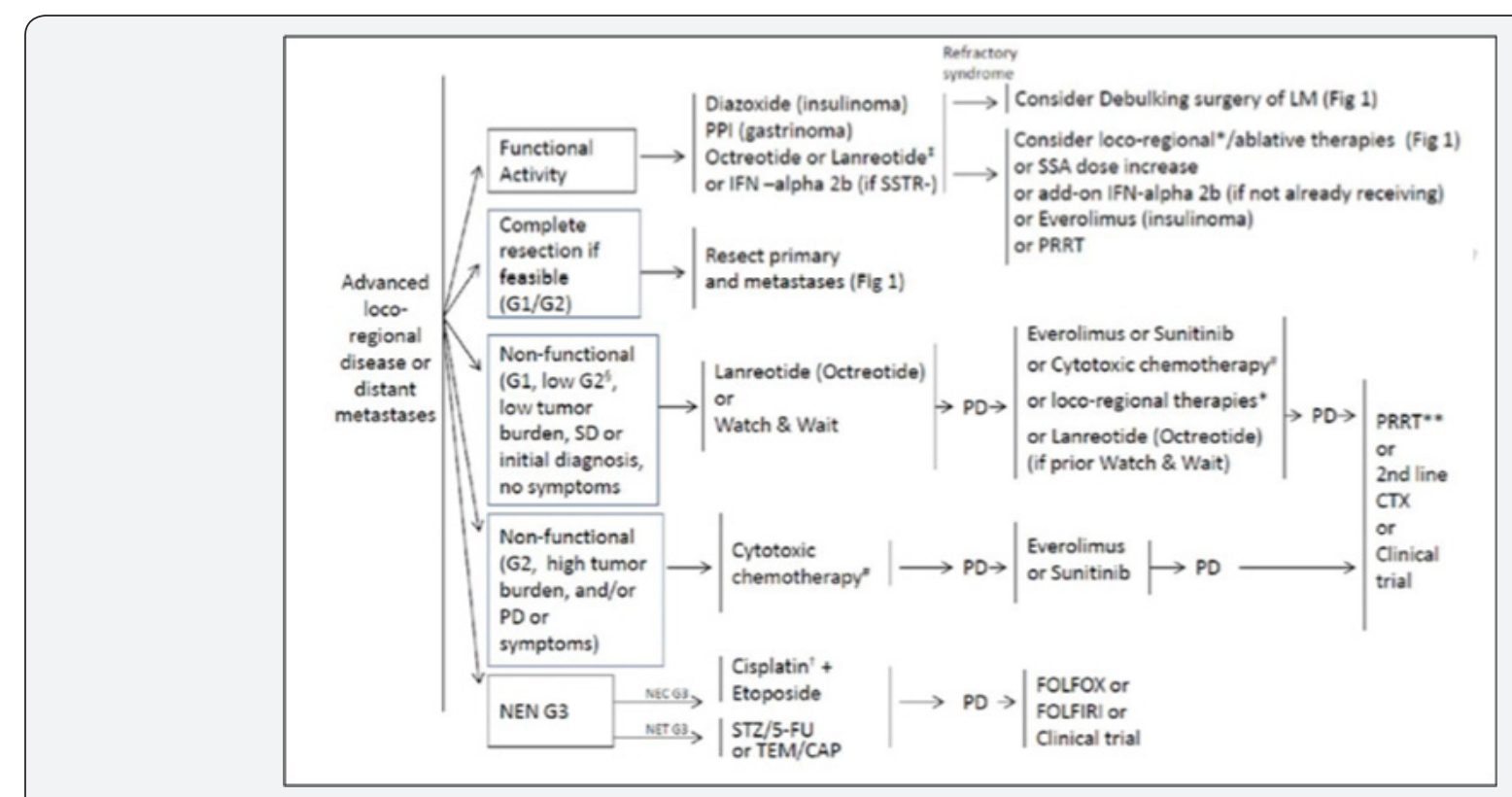

Figure 3: Algorithms proposed for patients with NETs of pancreatic origin and liver metastases. 2016 ENETS Guidelines.

Legend : PD progressive disease, SD stable disease, SSTR somatostatin receptor, SSA somatostatin analogs, CS carcinoid syndrome, PRRT peptide receptor radionuclide therapy, LM liver metastases, TEM/CAP temozolomide/capecitabine, STZ streptozotocin, 5-FU 5-fluorouracil. Similarly, for NETs of pancreatic origin treatment decisions are based on the same questions presented for (Figure 2) in reference to NETs of gastrointestinal origin.

The subdivision of G3 tumors into G3 NETs, well differentiated tumors, and G3 NECs, poorly differentiated tumors with only one treatment option based on platinumetoposide chemotherapy should be noted. Furthermore, we should underline that observation is less feasible in pancreatic tumors, that is, no active treatment in the setting of advanced disease after the new evidence available from the CLARINET trial, which included gastrointestinal and pancreatic tumors with a Ki as high as $10 \%$. Also, there may be a more importantrole for chemotherapy although with poor levels of evidence to define the best chemotherapy scheme in this context.

\section{Conclusion}

Interesting development is ongoing in the field of neuroendocrine tumors by cooperative groups and well designed clinical trials to answer questions about systemic, surgical or local liver ablative management. Working groups like NANETS, the American Group, or ENETS, the European Neuroendocrine Tumor Society, have pioneered the development of both diagnosis and treatment guidelines for this condition. ARGENTUM, the working group in our country, more humbly and with certain limitations, studies the local data and provides a chance for sharing experiences between our region (LATAM) with the leading centers in the world. Our great challenge for the future is to learn more about the best treatment sequence for this condition as well as the molecular characterization of different tumor subtypes, in order to optimize resources and take a step closer to precision medicine or personalized medicine in this field. A multidisciplinary approach together with the integration of the participating specialists, not only oncologists and surgeons, is the key to succeed and advance in research to offer patients with this condition the best treatment option.

\section{References}

1. O'Connor JM, Marmissolle F, Bestani C, Pesce V, Belli S, et al. (2014) Observational study of patients with gastroenteropancreatic and bronchial neuroendocrine tumors in Argentina: Results from the large database of a multidisciplinary group clinical multicenter study. Mol Clin Oncol 2(5): 673-684. 
2. Sorbye H, Welin S, Langer SW, Vestermark LW, Holt N, et al. (2013) Predictive and prognostic factors for treatment and survival in 305 patients with advanced gastrointestinal neuroendocrine carcinoma (WHO G3): the NORDIC NEC study. Ann Oncol 24(1): 152-160.

3. Milione M, Maisonneuve P, Spada F, Pellegrinelli A, Spaggiari P, et al. (2017) The Clinicopathologic Heterogeneity of Grade 3 Gastroenteropancreatic Neuroendocrine Neoplasms: Morphological Differentiation and Proliferation Identify Different Prognostic Categories. Neuroendocrinology 104(1): 85-93.

4. Kulke MH, Hörsch D, Caplin ME, Anthony LB, Bergsland E, et al. (2017) Telotristat Ethyl, a Tryptophan Hydroxylase Inhibitor for the Treatment of Carcinoid Syndrome. J Clin Oncol 35(1): 14-23.
5. Caplin ME, Pavel M, Ćwikła JB, Phan AT, Raderer M, et al. (2016) Anti-tumour effects of lanreotide for pancreatic and intestinal neuroendocrine tumours: the CLARINET open-label extension study. Endocr Relat Cancer 23(3): 191-199.

6. Niederle B, Pape UF, Costa F, Gross D, Kelestimur F, et al. (2016) ENETS Consensus Guidelines Update for Neuroendocrine Neoplasms of the Jejunum and Ileum. Neuroendocrinology 103(2): 125-138.

7. Pavel M, O’Toole D, Costa F, Capdevila J, Gross D, et al. (2016) ENETS Consensus Guidelines Update for the Management of Distant Metastatic Disease of Intestinal, Pancreatic, Bronchial Neuroendocrine Neoplasms (NEN) and NEN of Unknown Primary Site. Neuroendocrinology 103(2): 172-185.

\section{Your next submission with JuniperPublishers will reach you the below assets}

- Quality Editorial service

- Swift Peer Review

- Reprints availability

- E-prints Service

- Manuscript Podcast for convenient understanding

- Global attainment for your research

- Manuscript accessibility in different formats

( Pdf, E-pub, Full Text, audio)

- Unceasing customer service

Track the below URL for one-step submission https://juniperpublishers.com/online-submission.php 